\title{
Educación en línea y evaluación del aprendizaje: de lo presencial a lo virtual
}

\author{
Online education and learning assessment: From face-to-face to virtual
}

\section{Avaliação da educação e aprendizagem online: do presencial ao virtual}

\author{
Yasmín Ivette Jiménez Galán \\ Instituto Politécnico Nacional, México \\ yjimenezg@ipn.mx \\ https://orcid.org/0000-0003-0575-7283 \\ Josefina Hernández Jaime \\ Instituto Politécnico Nacional, México \\ johernandezja@ipn.mx \\ https://orcid.org/0000-0001-8299-3736 \\ Eduardo Rodríguez Flores \\ Instituto Politécnico Nacional, México \\ erodrigrezf@ipn.mx \\ https://orcid.org/0000-0003-0562-2282
}

\section{Resumen}

En el mes de marzo de 2020, la situación de contingencia sanitaria, a nivel mundial y en México particularmente, exigió nuevas formas de articular los procesos de enseñanza - aprendizaje para evitar la cancelación del ciclo escolar; para el Instituto Politécnico Nacional supuso aprovechar al máximo las tecnologías de la información y la comunicación. Para ello puso a la disposición de los docentes y alumnos recursos digitales didácticos, objetos de aprendizaje, cursos y tutoriales para el manejo de plataformas educativas como CLASSROOM, MOODLE, EDMODO, entre otras. Con lo que se pretendía que las clases presenciales fueran sustituidas por clases en línea.

Para conocer cuáles fueron las adaptaciones pedagógicas que realizaron los docentes para impartir sus cursos en línea y con la finalidad de analizar cuáles fueron los principales obstáculos que 


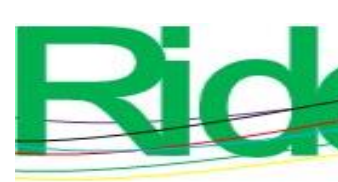

Revista Iberoamericana para la Investigación y el Desarrollo Educativo ISSN $2007-7467$

enfrentaron, y las formas de evaluación de los aprendizajes que implementaron, se diseñó esta investigación exploratoria - descriptiva que tuvo por objetivo explorar la percepción de 15 docentes relacionada con el diseño de sus clases en línea, durante marzo - julio del 2020. Los resultados muestran que el $13 \%$ considera que los alumnos en este esquema en línea están aprendiendo igual o más que en sus cursos presenciales y el $87 \%$ restante opina que los alumnos están aprendiendo menos porque no están acostumbrados a ser autodidáctas y tienen un aprendizaje conductivo dependiente del docente. Por otro lado, al preguntarles su percepción sobre qué les hizo falta durante este periodo de clases en línea, 47\% consideró que desarrollar más materiales didácticos digitales e interactivos, $27 \%$ consideró que desarrollar una planeación didáctica más efectiva, al $13 \%$ les hizo falta infraestructura porque en las sesiones mediante la plataforma Zoom se les caía el Internet; y 13\% enfatizó que les hizo falta más capacitación en herramientas de gestión de las TIC.

Se concluye que la evaluación del proceso de aprendizaje sigue siendo la mayor área de oportunidad para lograr elevar la calidad de la enseñanza - aprendizaje en línea. Asimismo, no existe prueba de que los docentes hicieran uso de la tecnología para diversificar sus prácticas evaluativas, al solamente trasladar lo que ya se tenía planeado en clases presenciales y montar los materiales y actividades en una plataforma educativa.

Palabras clave: aprendizaje, educación en línea, instrumentos de evaluación, plataformas educativas, proceso de evaluación.

\section{Abstract}

In the month of March 2020, the health contingency situation, worldwide and in Mexico particularly, demanded new ways of articulating the teaching-learning process to avoid the cancellation of the school year; for the National Polytechnique Institute it meant making the most of ICT. For this, it made educational digital resources, learning objects, courses and tutorials available to teachers and students for the management of educational platforms such as CLASSROOM, MOODLE, and EDMODO.

In order to find out what were the pedagogical adaptations that teachers made to teach their online courses and to analyze what were the main obstacles they faced, this exploratory - descriptive research was designed that aimed to explore the perception of 15 teachers related to the design of their online classes, during March - July 2020, and the forms of evaluation of the learning they implemented. The results show that $13 \%$ consider that students in this online scheme are learning the same or more than in their face-to-face courses and the remaining $87 \%$ believe that students 


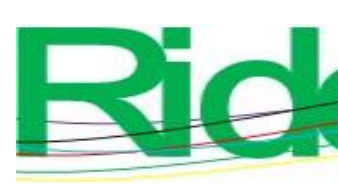

Revista Iberoamericana para la Investigación y el Desarrollo Educativo ISSN $2007-7467$

are learning less because students are not used to being self-taught and have a conductive learning, dependent on the teacher. Finally, when asked their perception about what they needed during this period of online classes, $47 \%$ considered that developing more digital and interactive teaching materials, $27 \%$ considered that developing more effective teaching planning, 13\% lacked infrastructure because in the platform Zoom sessions were dropping the internet; and 13\% emphasized that they needed more training in ICT management tools.

There is no evidence that teachers made use of technology to diversify their assessment practices, by only transferring what was already planned in face-to-face classes and uploading materials and activities on an educational platform; therefore the evaluation of the learning process continues to be the greatest area of opportunity to achieve raising the quality of online teaching - learning .

Keywords: learning, online education, evaluation instruments, educational platforms, evaluation process.

\section{Resumo}

Em março de 2020, a situação de contingência em saúde, mundial e principalmente no México, exigia novas formas de articular os processos de ensino-aprendizagem para evitar a extinção do ciclo escolar; Para o Instituto Politécnico Nacional, significou valorizar as tecnologias de informação e comunicação. Para isso, disponibilizou recursos digitais educacionais, objetos de aprendizagem, cursos e tutoriais a professores e alunos para o gerenciamento de plataformas educacionais como SALA DE AULA, MOODLE, EDMODO, entre outras. Com o que se pretendia que as aulas presenciais fossem substituídas por aulas online.

Com o objetivo de conhecer quais foram as adaptações pedagógicas que os professores fizeram para ministrar seus cursos online e para analisar quais foram os principais obstáculos que enfrentaram e as formas de avaliação da aprendizagem que implementaram, esta investigação exploratório-descritiva foi elaborada. teve como objetivo explorar a percepção de 15 professores em relação ao design das suas aulas online, durante o período de março a julho de 2020. Os resultados mostram que $13 \%$ consideram que os alunos neste regime online estão a aprender o mesmo ou mais do que no presencial cursos e os $87 \%$ restantes acreditam que os alunos estão aprendendo menos porque não estão acostumados a serem autodidatas e têm aprendizagem comportamental dependente do professor. Por outro lado, quando questionados sobre a percepção do que precisavam durante esse período de aulas online, 47\% consideraram que desenvolver materiais didáticos mais digitais e interativos, $27 \%$ consideraram que desenvolvendo um 


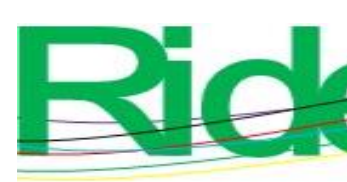

Revista Iberoamericana para la Investigación y el Desarrollo Educativo ISSN $2007-7467$

planejamento didático mais eficaz, $13 \%$ necessitavam de infraestrutura porque em as sessões por meio da plataforma Zoom largaram da Internet; e 13\% enfatizaram que precisam de mais treinamento em ferramentas de gestão de TIC.

Conclui-se que a avaliação do processo de aprendizagem continua sendo a maior área de oportunidade para se alcançar a elevação da qualidade do ensino - aprendizagem online. Da mesma forma, não há evidências de que os professores tenham feito uso da tecnologia para diversificar suas práticas avaliativas, apenas transferindo o que já estava planejado nas aulas presenciais e montando os materiais e atividades em uma plataforma educacional.

Palavras-chave: aprendizagem, educação online, instrumentos de avaliação, plataformas educacionais, processo de avaliação.

Fecha Recepción: Octubre 2020

Fecha Aceptación: Agosto 2021

\section{Introducción}

La sociedad del conocimiento y el avance de las TIC han influido fuertemente para que las instituciones de educación superior (IES) reformulen sus perfiles de egreso hacia profesionistas competentes para resolver las diversas situaciones problemáticas de su campo y hábiles en el manejo de las TIC (Alvarado, 2014; Bautista, Borges y Forés, 2006). Adicionalmente, las TIC han revolucionado las modalidades educativas presencial, semipresencial y en línea para brindar mayor cobertura a la población. Por ende, Alvarado (2014) aboga por que se extienda la visión de que las TIC, más que una herramienta de apoyo para la educación, sean consideradas como la oportunidad de potenciar la capacidad de las instituciones que promueven el conocimiento, de ahí que las IES hayan venido trabajando de manera paulatina en dicho objetivo.

Sin embargo, la crisis sanitaria por la covid-19 obligó al cambio drástico de clases presenciales a clases en línea, lo que aceleró la incorporación de las TIC en los procesos de enseñanza-aprendizaje para dar respuesta satisfactoria al contexto modificado. Dicha incorporación supone que tanto docentes como estudiantes desarrollen competencias digitales (Lazcano y Vilanova, 2017; Moreira y Delgadillo, 2014) para lograr que la enseñanza y el aprendizaje sean eficientes y efectivos, pues tales competencias les permitirán a los egresados insertase en el campo laboral con mayor facilidad (Alvarado, 2014).

No es aventurado asegurar que el impulso brindado por las TIC ha cobrado cada día mayor relevancia y presencia dentro del ámbito educativo, por lo que su utilización se vislumbra como algo permanente en los procesos de enseñanza-aprendizaje en la medida en que las instituciones y los hogares sean equipados con tecnología (software, hardware, aplicaciones, etc.). 


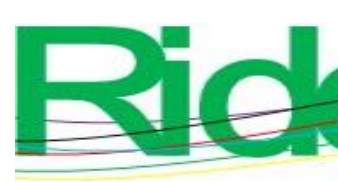

Revista Iberoamericana para la Investigación y el Desarrollo Educativo ISSN $2007-7467$

Concretamente, dentro de las ventajas de la educación en línea se enfatizan la superación de las barreras de distancia y de la rigidez de los horarios de clases, así como la comunicación asíncrona y el manejo de información más variada para lograr un objetivo o meta (Bautista, Borges y Forés, 2006; Moreira y Delgadillo, 2014; Ruiz, 2013).

No obstante todas las ventajas, también la educación en línea presenta desventajas tanto para estudiantes como para los docentes. En general, los alumnos enfrentan tres grandes desafíos con relación con las clases presenciales: la inflexibilidad instruccional que puede llegar a convertir los procesos de enseñanza-aprendizaje en un cúmulo de tareas con fechas de inicio y de entrega; una baja articulación entre la totalidad de los conocimientos adquiridos durante su formación académica; y una escasa sensación de logro a pesar de tener resultados parciales de cada actividad o proyecto entregado.

Por su parte, para los docentes que no están capacitados la educación en línea puede ser vivida como una experiencia abrumadora en la que todo el tiempo se está calificando tareas, actividades, prácticas o proyectos (Lazcano y Vilanova, 2017). En este sentido, y para los efectos de esta investigación, se podrían adoptar los siguientes referentes teóricos: a) la evaluación de los aprendizajes en la modalidad en línea es uno de los aspectos que ha representado mayores retos para los docentes y b) los docentes han tenido que modificar la forma en que venían dando las clases presenciales para su impartición en línea.

De acuerdo con Lazcano y Vilanova (2017), Álvarez y Álvarez (2012), Escudero (2010) y Bautista, Borges y Forés (2006), la calidad de la enseñanza de nivel superior es multifactorial debido a que intervienen el currículo, los programas de estudios, la metodología didáctica, los recursos, las instalaciones, el profesorado, etc. No obstante, Barberá (2006) enfatiza que concretamente existen tres aspectos que podrían representar un mayor potencial para la educación en línea: el ajuste sobre el contenido del aprendizaje, el desarrollo de trabajo colaborativo y la evaluación de los aprendizajes. Los tres aspectos están estrechamente interrelacionados, aunque el proceso de evaluación es el aglutinador de los procesos enseñanza-aprendizaje y, por lo tanto, del logro del desarrollo de las competencias específicas de cada asignatura. Es decir, de todos los factores sine qua non para lograr los perfiles de egreso profesionales de personas (competentes, autónomas, responsables, éticas y comprometidas con su desarrollo profesional, entre otros) es fundamental que los docentes diseñen un sistema de evaluación de los aprendizajes alineado y coherente con la malla curricular de las instituciones educativas. 


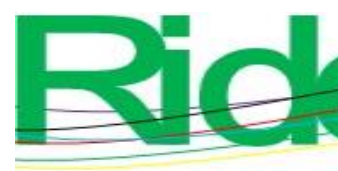

Revista Iberoamericana para la Investigación y el Desarrollo Educativo ISSN $2007-7467$

Así, la evaluación de los aprendizajes se convierte en el eje central de la enseñanza al ser un proceso obligado que permite establecer una armonía entre las competencias por desarrollar en los alumnos, las estrategias y los productos de aprendizaje que se solicitarán a lo largo del ciclo escolar, y tener un efecto proactivo en los estudiantes, pues les indica a priori lo que se espera que aprendan (Escudero, 2010; Lazcano y Vilanova, 2017). Recordemos que en la educación en línea no existe interacción cara a cara entre docentes y alumnos, por lo que la evaluación se convierte en el factor más importante para generar la automotivación de los alumnos por su formación (Anijovich y Cappelletti, 2017).

Para ello, la evaluación del aprendizaje en entornos virtuales exige que los docentes conozcan y comprendan desde el perfil de egreso, el mapa curricular, el modelo pedagógico hasta el programa de estudios, de forma tal que les permita tener una visión del conjunto de las asignaturas y del cómo cada una de ellas contribuye en la formación del estudiante. Dentro de este contexto, se entiende por e-evaluación al proceso de valoración del aprendizaje mediado por la tecnología que busca promover el desarrollo de las competencias propias de cada asignatura. Este debe basarse en objetivos claros y desarrollarse a través de una mediación pedagógica interactiva buscando la armonía del saber conocer, saber hacer, saber ser y estar para que los alumnos logren desempeñarse con éxito tanto en el ámbito escolar como en el laboral (Alvarado, 2014; Lazcano y Vilanova, 2017; Ruiz, 2013).

Resulta importante resaltar que la tecnología ha aportado por lo menos tres cambios y ventajas en la evaluación de los alumnos: evaluación automática con base en pruebas electrónicas tipo test mediante las cuales el alumno obtiene un resultado inmediato de su calificación; evaluación enciclopédica con base en trabajos individuales tipo ensayos, y evaluación colaborativa con base en foros de discusión o grupos de trabajo donde se puede establecer la coevaluación y se posibilita la oportunidad de evaluar el desempeño del trabajo en equipo (Barberá, 2006; Lazcano y Vilanova, 2017). En consecuencia, se puede realizar una valoración más completa de los estudiantes al evaluar no solo sus productos o resultados, sino los procesos realizados para llegar a dichos objetivos.

Si bien la tecnología permite optimizar y diversificar las prácticas evaluativas de los docentes de cursos en línea (Escudero, 2010; Ruiz, 2013), se reitera que estas deben estar contextualizadas y alineadas con el modelo educativo y las competencias por desarrollar en cada asignatura, por lo que cada evaluación debe responder a qué, para qué, cómo, cuándo y con qué evaluar (Anijovich y Cappelletti, 2017). 

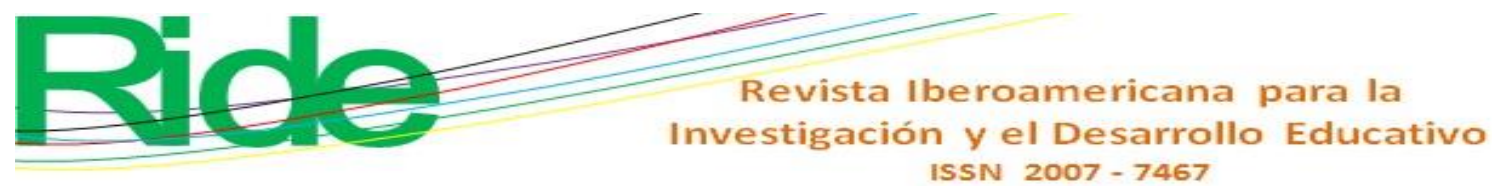

Por otro lado, algunos investigadores enfatizan que en ambientes virtuales de aprendizaje la evaluación se reconoce, pero no se practica con la multidimensionalidad que implica dicho concepto. De acuerdo con Barberá (2006), la evaluación tiene cuatro dimensiones que deben ser abordadas deliberadamente por el docente y practicadas por alumnos y docentes. Una primera dimensión es la evaluación "desde el aprendizaje", de forma tal que le sirva a los alumnos para conectar lo previamente aprendido en su trayectoria académica con los nuevos conocimientos; así, lo nuevo podría convertirse en significativo porque les permite establecer un andamiaje de conocimientos.

La segunda y tercera dimensión de la evaluación es "del aprendizaje" que permite valorar cómo se van desempeñando los alumnos, y "para el aprendizaje" cuya base primordial es la retroalimentación del desempeño tanto de los docentes como de los alumnos. Con la retroalimentación se puede llegar a establecer un diálogo docente-alumnos organizado alrededor de los contenidos temáticos y ofrecer ayuda ajustada a las necesidades individuales de los estudiantes para que puedan desarrollar las competencias propias de cada unidad de aprendizaje y, en conjunto, las de su perfil del egreso. Finalmente, la cuarta dimensión guarda relación con la evaluación "como aprendizaje", que coadyuva a los estudiantes a regular el propio aprendizaje para lograr los fines educativos (esta última dimensión solo la posibilitan algunos instrumentos o técnicas de evaluación).

Existe una gran variedad de estrategias e instrumentos de evaluación mediados por las TIC y centrados en el estudiante, que permiten a los docentes constatar sus avances a lo largo de la trayectoria académica; dentro de los más importantes para desarrollar competencias se podrían mencionar los siguientes: pruebas objetivas, desarrollo de proyectos, foros, wikis, portafolios de evidencias, rúbricas y listas de cotejo (Escudero, 2010; Lazcano y Vilanova, 2017). Particularmente, el portafolio de evidencias, las rúbricas y las listas de cotejo son reconocidas como las más potentes para regular el aprendizaje y promover la autonomía y la autodirección al motivar a los alumnos a ser responsables de su propio desarrollo.

Para que la evaluación en ambientes virtuales de aprendizaje no pierda su riqueza en la formación de los alumnos, se requiere que los docentes diseñen un modelo de evaluación y una metodología que incluya diversos tipos de instrumentos y técnicas. Adicionalmente, dado que a la evaluación formativa se le confiere un mayor peso en la ponderación de la calificación final del curso porque es en la que se basa la construcción de conocimientos nuevos y de aprendizajes significativos, el docente de cursos en línea deberá estar particularmente dispuesto a retroalimentar el desempeño de los alumnos. Esa retroalimentación deberá ser continua, clara, oportuna, suficiente 


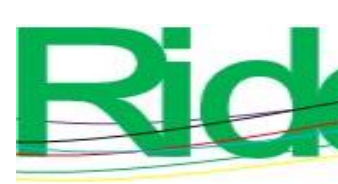

Revista Iberoamericana para la Investigación y el Desarrollo Educativo ISSN $2007-7467$

digitales didácticos, objetos de aprendizaje, y cursos y tutoriales para el manejo de plataformas educativas como Classroom, Moodle, Edmodo, entre otras. Con esto se pretendía que las clases presenciales fueran sustituidas por clases en línea.

De acuerdo con Torres, Prieto y López (2012), el buen uso de las TIC para la docencia es, sin duda, una opción ventajosa tanto para el profesorado como para el alumnado; no obstante, el desarrollo de las competencias digitales y la facilidad en el manejo de las herramientas requiere de un periodo de adaptación, el cual no se tuvo. Por lo tanto, para los docentes del IPN esto representó variados retos que incluyeron desde la adaptación de sus recursos didácticos hasta la modificación de sus prácticas evaluativas.

Por ello, el objetivo de esta investigación fue explorar la percepción de los docentes del IPN en torno al diseño de sus clases en línea durante marzo-julio de 2020, así como las estrategias que implementaron para evaluar los aprendizajes.

\section{Materiales y métodos}

El tipo de estudio fue el exploratorio-descriptivo. Exploratorio porque el problema de investigación es reciente y poco explorado, ya que fue ocasionado por la covid-19, lo que obligó a articular cursos en línea que antes eran presenciales. Además, es descriptivo porque se buscó contribuir con los resultados a la masa crítica de la problemática de la e-evaluación detallando las dificultades, retos e interpretación de la cotidianidad (Tamayo, 2006) que enfrentaron los docentes para estructurar sus cursos en línea. Asimismo, se basó en un diseño no experimental transeccional (Hernández, Fernández y Baptista, 2014) efectuado en el mes de junio de 2020.

El diseño de la investigación fue cualitativo porque se trató de entender, desde la perspectiva de los docentes, cómo articularon y por qué decidieron sus prácticas evaluativas para sus respectivos cursos. El método de recolección de la información fue la entrevista, por lo que se desarrolló un guion de entrevista semiestructurada que permitiera tanto a los docentes como a los investigadores indagar más a fondo cuestiones relacionadas con los cursos respectivos.

Por tratarse de un estudio exploratorio, el muestreo fue no estadístico por conveniencia, dada la accesibilidad y la voluntad de los docentes para ser incluidos en la investigación; asimismo, se consideró importante contar con la percepción de docentes de los cuatro departamentos académicos de una unidad académica de nivel superior del IPN.

El guion de entrevista se diseñó en dos secciones: la primera fue para recolectar información general de los docentes encuestados; la segunda (ver tabla 1) estuvo basada en las dimensiones de la evaluación propuestas por Barberá (2006). El guion de entrevista se envió por correo electrónico 

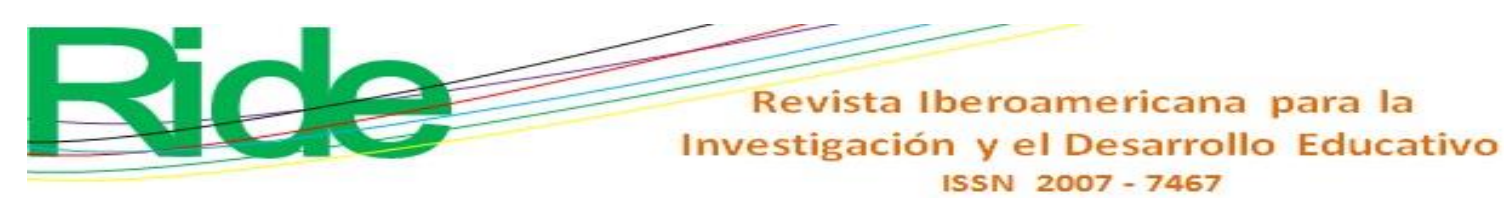

La dinámica de las clases inicia con la planeación del tema, checo si el material que tengo es el adecuado; si no, lo modifico para hacerlo más concreto y lo lleno de ejemplos. Las sesiones presenciales las programo por medio de la plataforma Zoom y los invito por Facebook, veo cuántos ya la vieron. Las sesiones las divido: cinco minutos saludos, diez minutos preguto cómo están, qué hicieron, cuáles son sus planes, cómo les va, que hablen todos, que sientan confianza y que son escuchados. Para el tema generalmente comparto pantalla con las presentaciones que son máximo de 10 o 12 diapositivas. Las sesiones son programadas, dos a la semana.

Otro docente explicó:

En la plataforma Classroom estructuré los temas, puse los ejercicios; doy sesiones por medio de la plataforma Zoom, pero son sesiones que sirven para explicar el contenido, no les resuelvo los ejercicios porque se pretende que ellos aprendan. Los ejercicios contestados los suben a la plaraforma y entonces ya los retroalimento $(14 / 15)$.

El método de enseñanza-aprendizaje que mayormente siguieron los docentes fue el deductivo y el desarrollo de proyectos (67\% de los entrevistados). Respecto al tipo de evidencias de aprendizaje que utilizaron, $60 \%$ de los docentes entrevistados utlizaron por lo menos cuatro tipos: cuestionarios, proyecto o problemarios, prácticas y exposición de algún contenido temático; $33 \%$ adicionalmente incluyó examen de conocimientos y $7 \%$ solo evaluó a sus alumnos con el desarrollo de un proyecto. En relación con los instrumentos de evaluación que utilizaron, solo 33 \% dijo haber utilizado rúbricas y $67 \%$ listas de cotejo y examen de conocimientos con retroalimentación automática a través de la plataforma. De los cinco docentes que utilizaron rúbricas, solo tres refirieron que sus alumnos sí las conocían de antemano.

Los medios empleados para retroalimentar el desempeño o las dudas de los alumnos fueron, principalmente, la plataforma elegida al calificar las actividades o tareas (53\%), mediante correo electrónico y plataforma (27 \%), y el restante $20 \%$ utilizó, además de la plataforma y el correo eléctrónico, aplicaciones de mensajería instantánea como Whattsapp o Messenger de Facebook. Es decir, el desempeño se retroalimentó a través de la plataforma y las dudas fueron atendidas por correo o mensajería.

Para explorar la percepción de los docentes entrevistados relacionada con la evaluación de los aprendizajes, se les preguntó por la modificación que hicieron sobre la marcha al cambiar de un esquema presencial a uno en línea, y por el grado de satisfacción que sentían por dichos cambios; 


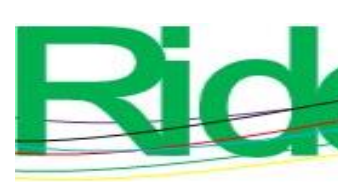

Revista Iberoamericana para la Investigación y el Desarrollo Educativo ISSN 2007 - 7467

es decir, su percepción de la pertinencia de dichas modificaciones para evaluar el aprendizaje de sus alumnos. En la tabla 3 se pueden apreciar las respuestas de los docentes.

Tabla 3. Formas de evaluación del aprendizaje

\begin{tabular}{|c|c|c|}
\hline N. ${ }^{\circ}$ & $\begin{array}{l}\text { Del } 1 \text { al } 10 \text { (donde } 1 \text { es poco y } 10 \text { es } \\
\text { mucho), ¿qué tanto modificó su forma de } \\
\text { evaluar a sus estudiantes? }\end{array}$ & $\begin{array}{l}\text { Del } 1 \text { al } 10 \text { (donde } 1 \text { es poco y } 10 \text { es } \\
\text { mucho), ¿qué tan satisfecho se siente con la } \\
\text { forma que implementó para evaluar a sus } \\
\text { estudiantes? }\end{array}$ \\
\hline 1 & $\begin{array}{l}\text { 4 / Aunque traté de apegarme a lo del } \\
\text { principio, las prácticas disminuyeron en } \\
\text { profundidad, pero las estructuré mejor; no } \\
\text { voy a hacer examen. }\end{array}$ & $\begin{array}{l}6 \text { / Por dos razones: } 1 \text {. Falta de tiempo, no } \\
\text { puedo tener más contacto, lo noto en las } \\
\text { dudas que expresan mis alumnos por la } \\
\text { plataforma Zoom. } 2 \text {. Las rúbricas son muy } \\
\text { puntuales. }\end{array}$ \\
\hline 2 & 2 / No apliqué exámenes semanales. & $\begin{array}{l}10 \text { / Bien, porque siento que mi forma de } \\
\text { trabajo no se modificó, por lo que los } \\
\text { alumnos no se están sorprendiendo. }\end{array}$ \\
\hline 3 & $\begin{array}{l}3 \text { / Cambié la estrategia, ahora tengo que } \\
\text { dejar tareas; en presencial no dejaba } \\
\text { tareas, en el salón se hacía todo. }\end{array}$ & $\begin{array}{l}7 \text { / Porque no tengo experiencia docente, los } \\
\text { estudiantes proponen cambios y no los } \\
\text { acepto. De momento no podría manejarlos. }\end{array}$ \\
\hline 4 & $\begin{array}{l}5 \text { / No he revisado el segundo entregable } \\
\text { del proyecto empresa, la presentación de } \\
\text { ellos no es lo mismo, no les puedo evaluar } \\
\text { el desarrollo de competencias directivas. }\end{array}$ & $\begin{array}{l}8 \text { / Soy muy flexible, quizá demasiado. } \\
\text { Están en cuarto nivel y siento que necesitan } \\
\text { mayor presión... }\end{array}$ \\
\hline 5 & $\begin{array}{l}2 \text { / Las evaluaciones y criterios son los } \\
\text { mismos. El examen se les mandó y fue a } \\
\text { libro abierto, el control fue diferente. Es } \\
\text { muy complicado resolver las dudas de } \\
\text { forma inmediata. }\end{array}$ & $\begin{array}{l}10 \text { / Creo que en comparación con la forma } \\
\text { presencial, me mantengo dentro de lo que se } \\
\text { pretende desarrollar en los estudiantes. }\end{array}$ \\
\hline 6 & $\begin{array}{l}\text { 1 / Se siguió con lo mismo, el curso se } \\
\text { había planeado para que los alumnos } \\
\text { mostraran un proyecto final por } \\
\text { micromódulos. Como no voy a poder ver } \\
\text { lo que hicieron agregué una práctica. }\end{array}$ & $\begin{array}{l}9 \text { / Primera vez que utilizo Edmodo, me } \\
\text { siento muy satisfecho porque todo está } \\
\text { concentrado y a la mano. Me facilitó la vida. }\end{array}$ \\
\hline 7 & $\begin{array}{l}3 \text { / Como se evalúa por proyectos no fue } \\
\text { mucho el cambio. }\end{array}$ & $\begin{array}{l}8 \text { / Porque me he dado cuenta que la clase } \\
\text { queda grabada en video y ellos pueden } \\
\text { consultarla continuamente. Si ha } \\
\text { funcionado, los ejercicios los están haciendo } \\
\text { muy bien. Me sorprendió gratamente que } \\
\text { algunos alumnos estaban aprendiendo más } \\
\text { por los videos. }\end{array}$ \\
\hline 8 & $\begin{array}{l}7 \text { / En la modalidad presencial era muy } \\
\text { estricta con tiempos. Yo evaluaba } \\
\text { conocimiento, desempeño y actitud. }\end{array}$ & $\begin{array}{l}5 \text { / Porque al interactuar con ellos califico su } \\
\text { conocimento, pero así no sé si ellos están } \\
\text { haciendo las tareas y los ejercicios. Se los }\end{array}$ \\
\hline
\end{tabular}




\begin{tabular}{|l|l|l|}
\hline & $\begin{array}{l}\text { Ahora diseñé actividades cortas, los } \\
\text { tiempos de entrega no son tan } \\
\text { importantes. Las participaciones tienen } \\
\text { menor peso que en presencial. }\end{array}$ & $\begin{array}{l}\text { pido a mano para checar que ellos lo hagan, } \\
\text { pero no lo puedo asegurar. }\end{array}$ \\
\hline 9 & $\begin{array}{l}\text { 7 / La UA está pensada para que sea } \\
\text { presencial, por lo que se cambiaron las } \\
\text { sesiones y se eliminaron los temas de } \\
\text { autoestima porque en clases en línea no se } \\
\text { pueden valorar. }\end{array}$ & $\begin{array}{l}\text { 4 / Porque he tenido que improvizar, a pesar } \\
\text { de que he tomado cursos, creo que no son } \\
\text { suficientes, nunca se pensó que algo así } \\
\text { pudiera suceder. }\end{array}$ \\
\hline 10 & $\begin{array}{l}5 \text { / No he aplicado exámenes, la } \\
\text { retroalimentación no es la misma. }\end{array}$ & $\begin{array}{l}\text { 7 / Porque considero que hay aspectos que } \\
\text { no se tenían contemplados. Esto nos agarró } \\
\text { sobre la marcha, y pues ni modo... tuvimos } \\
\text { que hacerlo. }\end{array}$ \\
\hline 11 & $\begin{array}{l}\text { 2/ Por la asistencia, ahorita no la tomo en } \\
\text { cuenta. }\end{array}$ & $\begin{array}{l}\text { 6/ Porque en lo presencial yo puedo valorar } \\
\text { cuando trabajan en equipo, ahorita no sé si } \\
\text { todos hayan trabajado. }\end{array}$ \\
\hline 12 & $\begin{array}{l}8 \text { / No hay examen. El examen valía } 50 \% \\
\text { y ahora evalúo con prácticas. }\end{array}$ & $\begin{array}{l}\text { 8 / Por lo del examen. } \\
\text { puntos de su calificación. }\end{array}$ \\
\hline 13 & $\begin{array}{l}\text { 2 / Tuve que buscar videos en Youtube } \\
\text { para anexarlos a las prácticas. }\end{array}$ & $\begin{array}{l}\text { 8 / Por lo del examen que tuve que } \\
\text { modificar. Lo demás siguió igual. }\end{array}$ \\
\hline 14 & $\begin{array}{l}\text { 2 / Me siento altamente insatisfecha, la UA } \\
\text { requiere que esté supervisando los ejercicios } \\
\text { y les resuelva dudas específicas, no lo he } \\
\text { podido hacer a distancia por falta de tiempo. } \\
\text { Me consume muchísimo tiempo preparar el } \\
\text { material. }\end{array}$ \\
\hline 15 & $\begin{array}{l}\text { 9 / Bien, porque siento que mi forma de } \\
\text { trabajo no se modificó mucho. Los alumnos } \\
\text { ya estaban acostumbrados. }\end{array}$ \\
\hline
\end{tabular}

\section{Fuente: Elaboración propia}

Un aspecto muy importante que se abordó en la entrevista fue la opinión de los docentes acerca del nivel de aprendizaje de los alumnos. Al respecto, $13 \%$ considera que los alumnos en este esquema en línea están aprendiendo igual o más: "Un diez, porque de manera presencial se les complica asistir, son jóvenes que ya trabajan; esto los está obligando a ser más autodidactas. Se están responsabilizando por su educación" (6/15); "un nueve, aunque tengo calificaciones más dispersas. En la modalidad presencial el promedio es siete. Ahora el promedio sigue siendo siete, pero hay unos de ocho o nueve, y los más bajos son de cinco o cuatro" (2/15). El 87 \% restante opina que los alumnos están aprendiendo menos: "Yo diría que un siete, porque no sé si pongan atención, sé que asisten porque aparecen conectados. Les hago preguntas de teoría a través de Kahoot, pero la interacción es baja. No tengo la certeza de que estén atendiendo y entendiendo lo 


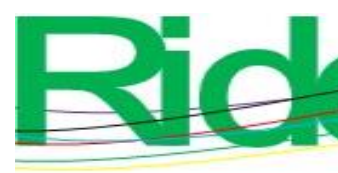

Revista Iberoamericana para la Investigación y el Desarrollo Educativo ISSN $2007-7467$

Esto demuestra que los docentes no están conscientes de la importancia de contextualizar sus prácticas evaluativas, ya que solamente $33 \%$ manifestó una notoria insatisfacción asignándole una calificación reprobatoria a dichas prácticas. En tal sentido, la mayor satisfacción que sienten en relación con la evaluación se refiere a la automatización del proceso gracias a las TIC.

El escaso reconocimiento por parte de los docentes de que el proceso de evaluación no se llevó a cabo como un proceso multidimensional (Barberá, 2006; Lazcano y Vilanova, 2017) podría repercutir en la pérdida de integralidad en la formación de los estudiantes, pero sobre todo en la nula innovación que este requiere para que los estudiantes reflexionen "desde el aprendizaje" para lograr su autonomía.

La información obtenida evidencia que existe confusión entre los docentes sobre la educación a distancia y en línea, lo que generó una discrepancia en los cursos, ya que unos fueron impartidos por Moodle asíncronamente y otros mediante videoconferencias (síncronos). Esto podría provocar discrepancias en la calidad de los aprendizajes, aunque el resultado final solo se podrá valorar en su completa magnitud a medida que los alumnos sigan avanzando en su trayectoria académica.

Aun así, el aprendizaje formativo fue atendido mediante las TIC de forma adecuada, puesto que todos de los docentes brindaron retroalimentación, como mínimo, mediante mensajes a través de la plataforma educativa elegida y más de la mitad utilizó adicionalmente el correo electrónico o aplicaciones de mensajería instantánea para resolver dudas de los alumnos.

Un tema puesto a discusión fue si el aprendizaje en línea superó o igualó al conseguido en la modalidad presencial, lo que generó división entre los docentes entrevistados. La premisa teórica, de acuerdo con Moreira y Delgadillo (2014), era que los docentes acostumbrados a las clases presenciales opinaran que el aprendizaje en línea es menor por el escaso contacto que se tiene con los alumnos y porque la experiencia en modalidades presenciales podría influir en su percepción; en este sentido, resultó sorprendente que $13 \%$ coincidiera en que el aprendizaje en línea es mejor porque los alumnos tienen a su disposición los materiales y videos para cuando quisieran consultarlos y seguir aprendiendo. 


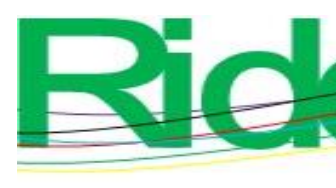

Revista Iberoamericana para la Investigación y el Desarrollo Educativo ISSN $2007-7467$

\section{Conclusiones}

Es importante enfatizar que el alcance de una investigación exploratoria sirve para generar hipótesis que deberán ser probadas posteriormente mediante investigaciones de mayor alcance. En este sentido, derivadas de los resultados de esta investigación, se pueden emitir tres hipótesis. La primera es que "la capacitación que requieren los docentes para articular sus cursos en línea debe estar enfocada en tres aspectos pedagógicos: el desarrollo de material didáctico interactivo, adecuación de los contenidos temáticos y principalmente en el desarrollo de un modelo de evaluación pertinente a las clases en línea". Aunque se reconoce la gran utilidad de las TIC para optimizar el proceso por medio de la evaluación automática, serán los docentes los que alineen las competencias pretendidas con las actividades, los productos y la retroalimentación proporcionada al desempeño de sus alumnos. Los resultados de esta investigación exploratoria indican que la evaluación del proceso de aprendizaje sigue siendo un área de oportunidad para lograr elevar la calidad de la enseñanza-aprendizaje en línea. Asimismo, no existe prueba de que los docentes emplearan la tecnología para diversificar sus prácticas evaluativas si solo convirtieron lo que tenían planeado para las clases presenciales en materiales y actividades digitales.

Una segunda hipótesis sería que "la evaluación del aprendizaje necesita adecuarse al entorno virtual", pues si continúa la contingencia sanitaria, para posteriores cursos las instituciones educativas tendrán que generar procesos de mayor reflexión sobre las implicaciones de los escasos cambios en las prácticas evaluativas de los docentes, toda vez que lo que funciona para evaluar de manera presencial no resulta tan eficiente de manera virtual.

Finalmente, la tercera hipótesis sería que "los cursos en línea logran aprendizajes de igual calidad que los cursos presenciales", siempre que docentes y alumnos cubran un perfil y se logre un cambio en los paradigmas de la enseñanza-aprendizaje.

\section{Futuras líneas de investigación}

Derivado de esta investigación, se vislumbran por lo menos tres líneas de investigación que resultan de alto interés para la comunidad educativa:

1. Análisis del perfil del docente en línea: Esto permitirá determinar cuáles son las competencias digitales que los docentes necesitan según los requerimientos del contexto donde laboran. Incluso esta línea de investigación abrirá el camino hacia los cursos de formación y capacitación profesional de los docentes. 

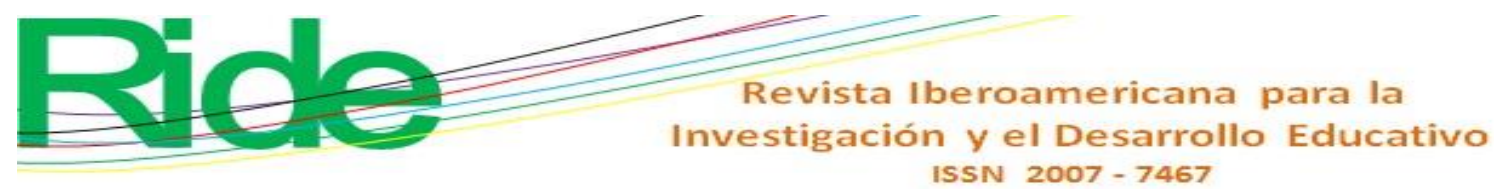

2. El proceso de evaluación de los aprendizajes en la educación en línea: Esto servirá para ahondar en la problemática que enfrentan los docentes en la conceptualización, el desarrollo y utilización de variados instrumentos y las implicaciones de sus prácticas evaluativas para lograr aprendizajes de calidad.

3. Creatividad e innovación en la migración de modalidades presenciales a modalidades en línea: Indudablemente que la educación en línea no puede ser un continuo de las estrategias de enseñanza-aprendizaje presenciales, por lo que se requiere del desarrollo de estudios que permitan correlacionar la creatividad y la innovación con el uso efectivo de la tecnología para adaptar o diseñar nuevas estrategias.

\section{Referencias}

Alvarado, M. (2014). Retroalimentación en educación en línea: una estrategia para la construcción del conocimiento. Revista Iberoamericana de Educación a Distancia, 17(2), 59-73. Recuperado de http://revistas.uned.es/index.php/ried/article/view/12678/11873

Álvarez, G. y Álvarez, G. (2012). Análisis de ambientes virtuales de aprendizaje desde una propuesta semiótico integral. Revista Electrónica de Investigación Educativa, 14(2), 73-86.

Anijovich, R. y Cappelletti, G. (2017). La evaluación como oportunidad. Buenos Aires, Argentina: Paidós.

Barberá, E. (2006). Aportaciones de la tecnología a la e-evaluación. Revista de Educación a Distancia, M6, 1-13. Recuperado de https://revistas.um.es/red/article/view/24301/23641

Bautista, G., Borges, F. y Forés, A. (2006). Didáctica universitaria en entornos virtuales de enseñanza-aprendizaje. Madrid, España. Narcea.

Escudero, T. (2010). Sin tópicos ni malos entendidos. Fundamentos y pautas para una práctica evaluadora de calidad en la enseñanza universitaria. Documento no. 9. Colección Documentos del Instituto de Ciencias de la Educación. Zaragoza, España. Recuperado de https://ice.unizar.es/sites/ice.unizar.es/files/users/leteo/publicaciones/doc09v2.pdf

Hernández, R., Fernández, C. y Baptista, P. (2014). Metodología de la investigación (6. ${ }^{a}$ ed.). México, D. F.: Mc Graw Hill, Educación.

Jaramillo, P. (2005). Uso de tecnologías de la Información en el aula: ¿qué saben hacer los niños con el computador? Revista de Estudios Sociales, 20, 27-44. Recuperado de http://www.scielo.org.co/scielo.php?script=sci_arttext\&pid=S0123-885X2005000100003 

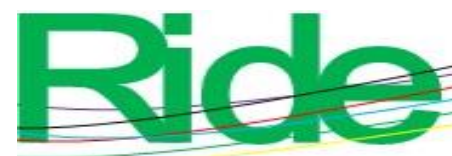

Revista Iberoamericana para la Investigación y el Desarrollo Educativo ISSN $2007-7467$

Lazcano, L. y Vilanova, G. (2017). Instrumentos de evaluación de aprendizaje en entornos virtuales. Perspectiva de estudiantes y aportes docentes. Informe Científico Técnico UNPA 9(1), 1- 36. Recuperado de https://dialnet.unirioja.es/servlet/articulo?codigo=5919087

Moreira, C. y Delgadillo, B. (2014). La virtualidad en los procesos educativos: reflexiones teóricas desde su implementación. Tecnología en Marcha, 28(1), 121-129. Recuperado de https://www.scielo.sa.cr/scielo.php?pid=S0379-

39822015000100121\&script=sci_abstract\&tlng=es

Quezada, R. (2006). Evaluación del aprendizaje en la educación a distancia “en línea”. Revista de Educación a Distancia, M6, 1-15. Recuperado de https://digitum.um.es/digitum/bitstream/10201/75321/1/02.pdf

Ruiz, Y. (2013) Evaluación de las competencias genéricas en la universidad. Estudio comparativo entre un entorno b-learning y presencial (tesis doctoral). Universidad Complutense de Madrid. Recuperado de https://eprints.ucm.es/24008/1/T35050.pdf

Tamayo, M. (2006). El proceso de la investigación científica (5. a ed.). México, D. F.: Limusa.

Torres, L., Prieto, E. y López, L. (2012). Entornos virtuales de enseñanza-aprendizaje. Evaluación del uso de las herramientas virtuales en el master de educación para el desarrollo. Edutec, 39, Recuperado de https://www.edutec.es/revista/index.php/edutec-e/article/view/376/113 


\begin{tabular}{|c|c|}
\hline Rol de Contribución & Autor (es) \\
\hline Conceptualización & Yasmín Ivette Jiménez Galán \\
\hline Metodología & $\begin{array}{l}\text { Yasmín Ivette Jiménez Galán, Josefina Hernández Jaime } \\
\text { (igual) }\end{array}$ \\
\hline Software & NO APLICA \\
\hline Validación & $\begin{array}{l}\text { Yasmín Ivette Jiménez Galán, Josefina Hernández Jaime } \\
\text { (igual) }\end{array}$ \\
\hline Análisis Formal & $\begin{array}{l}\text { Yasmín Ivette Jiménez Galán, Josefina Hernández Jaime } \\
\text { (igual) }\end{array}$ \\
\hline Investigación & $\begin{array}{l}\text { Yasmín Ivette Jiménez Galán (principal) y Eduardo Rodríguez } \\
\text { Flores (apoya) }\end{array}$ \\
\hline Recursos & $\begin{array}{l}\text { Yasmín Ivette Jiménez Galán (principal), Josefina Hernández } \\
\text { Jaime y Eduardo Rodríguez Flores (apoyan) }\end{array}$ \\
\hline Curación de datos & $\begin{array}{l}\text { Yasmín Ivette Jiménez Galán (principal) y Eduardo Rodríguez } \\
\text { Flores (apoya) }\end{array}$ \\
\hline $\begin{array}{l}\text { Escritura - Preparación del } \\
\text { borrador original }\end{array}$ & $\begin{array}{l}\text { Yasmín Ivette Jiménez Galán (principal), Josefina Hernández } \\
\text { Jaime y Eduardo Rodríguez Flores (apoyan) }\end{array}$ \\
\hline $\begin{array}{l}\text { Escritura - Revisión y } \\
\text { edición }\end{array}$ & $\begin{array}{l}\text { Yasmín Ivette Jiménez Galán, Josefina Hernández Jaime } \\
\text { (igual) }\end{array}$ \\
\hline Visualización & $\begin{array}{l}\text { Yasmín Ivette Jiménez Galán, Josefina Hernández Jaime } \\
\text { (igual) }\end{array}$ \\
\hline Supervisión & Yasmín Ivette Jiménez Galán \\
\hline Administración de Proyectos & Yasmín Ivette Jiménez Galán \\
\hline Adquisición de fondos & NO APLICA \\
\hline
\end{tabular}

